\title{
The dynamics of hierarchical age-structured populations
}

\author{
J. M. Cushing \\ Department of Mathematics, Interdisciplinary Program on Applied Mathematics, Building 89, \\ University of Arizona, Tucson, AZ 85721, USA
}

Received 16 March 1993; received in revised form 25 October 1993

\begin{abstract}
An age-structured population is considered in which the birth and death rates of an individual of age $a$ is a function of the density of individuals older and/or younger than $a$. An existence/uniqueness theorem is proved for the McKendrick equation that governs the dynamics of the age distribution function. This proof shows how a decoupled ordinary differential equation for the total population size can be derived. This result makes a study of the population's asymptotic dynamics (indeed, often its global asymptotic dynamics) mathematically tractable. Several applications to models for intra-specific competition and predation are given.
\end{abstract}

Key words: Age-structured population dynamics - McKendrick equations Hierarchical models - Existence/uniqueness - Asymptotic dynamics - Global stability - Intra-specific competition - Cannibalism

\section{Introduction}

The prototypical model for a self regulating population is the famous logistic equation. The biological assumptions on which this equation is based are highly qualitative and its ability to provide an adequate understanding of the dynamics of most populations is limited. For a discussion of the shortcomings of the logistic equation see [21]. Phenomena such as intra-specific competition or predation which can serve to regulate unrestricted exponential growth take place at the level of the individual organism. Therefore, because they in effect treat all individuals as identical, simple ordinary differential equation models for gross population-level statistics such as total population size, of which the logistic equation is just one example, cannot be expected to provide an adequate account of the dynamics of most biological populations unless they are enhanced and supported by individual-level sub-models for birth and death rates. This means that, to some extent at least, differences between individual organisms must be taken into account. One way to do this is to make use of the modeling techniques of "structured" population dynamics (see [25] for a thorough account of this methodology). 
Unfortunately, however, the equations of structured population dynamics involve rather complicated nonlinear integro-partial differential equations which, moreover, often have accompanying nonlinear integro-boundary conditions. Such model equations are likely to be very difficult to analyze, of course, and the goal of understanding the asymptotic dynamics of the total population from a knowledge of individual-level properties can be mathematically intractable. In general one can usually hope to obtain only equilibrium existence and local stability results by standard linearization methods (if not rigorously, at least formally). However, certain subclasses of structured models, i.e. models with certain restrictive assumptions on the nonlinearities or the age-specific effects on the vital rates, can often be analyzed by means of special techniques and tricks.

For example, certain kinds of age-structured models whose death rates depend on chronological age and on total population size in an additively separable manner have been analyzed rather thoroughly by techniques that derive decoupled equations for total population size and the (normalized) age distribution [2], [25], [27]. Another example is the "linear chain trick" by means of which a system of ordinary differential equations for total population size and certain other weighted integrals of the population age distribution are derived under the assumption that the age specific birth rate is described by means of certain kinds of distributions of the form $a^{p} e^{-q a}$ [16], [25].

In this paper we will consider a general class of nonlinear age-structured model equations for which it is possible to derive a decoupled ordinary differential equation for the population level dynamics. As a result of this method and the theorems presented below, a thorough analysis of the asymptotic dynamics of both the total population size and the age distribution of the population can often be carried out. This class of models is based upon a specialized class of sub-models which describes the nonlinear dependence of age-specific birth and death rates on population density. Specifically, for an individual of age $a$ these vital rates are assumed to depend on the sizes of the classes of individuals older and/or younger than $a$ (see (5) below). This assumption is motivated by populations in which there is a ranking based on chronological age that affects an individual's birth and death rates through, for example, access to food resources, desirable habitat or shelter, mates, nesting sites, etc.

For a study of the importance of hierarchical rankings in biological populations (not necessarily based upon age) see [21]. Attention will be restricted here to hierarchical rankings based on chronological age. Other ranking characteristics are important in the biological world, relative individual body size being one notable example [12], [21], [32]. The methods developed below can be extended to certain similar kinds of size-structured models; see for example [5]. Nonetheless, an age-structured model might quite adequately describe the dynamics of a population of individuals whose body size (or any other relevant ranking characteristic) strongly correlates with chronological age, as is often the case.

In Sect. 2 the model equations are described and certain technical assumptions and definitions are given. An existence/uniqueness result for a solution of these equations is given by Theorem 2 in Sect. 3 (and proved in Appendix B). The basic existence/uniqueness results for age-structured population models of Gurtin and MacCamy [15] and Tucker and Zimmerman [29] do not apply to our Problem 2 below because of the nature of the nonlinear density dependence in (5) (although their proofs could probably be adapted to apply to our model equations). The results of Webb [31] do apply to our equations. However, we choose to give an 
independent theorem and proof, in a different mathematical setting. We do this in order to take full advantage of the specialized nonlinearities in the age hierarchical model in such a way as to accomplish our primary goal of deriving a decoupled dynamical equation for total population size. Moreover, our approach and proof will yield a global existence theorem, as opposed to the usual local existence theorem. In Sect. 4 the special case of autonomous models is considered in more detail. Theorem 3 describes the global asymptotic dynamics of both total population size and the age distribution function for autonomous age hierarchical models. Several applications to questions concerning different kinds of intra-specific competition (viz. contest vs. scramble) and intra-specific predation (cannibalism) are given in Sect. 5. We emphasize that in all of these applications the methods developed here allow us to obtain a global description of the asymptotic dynamics.

\section{Model equations}

The dynamics of an age-structured population can be described by a density function $\rho=\rho(t, a) \geqq 0$ where $t$ is time and $a$ is chronological age. Thus, $\int_{a_{1}}^{a_{2}} \rho(t, a) d a$ is the number of individuals in the population between ages $a_{1}$ and $a_{2}$. This density function satisfies the equations

$$
\begin{gathered}
\lim _{h \rightarrow 0} \frac{\rho(t+h, a+h)-\rho(t, a)}{h}+\delta \rho(t, a)=0, \quad a>0, t>0 \\
\rho(t, 0)=\int_{0}^{+\infty} \beta \rho(t, a) d a, \quad t>0 \\
\rho(0, a)=\varphi(a), \quad a \geqq 0
\end{gathered}
$$

where $\beta$ and $\delta$ are birth and death rates and $\varphi$ is the initial age distribution [15], [20], [25]. These are often referred to as the McKendrick equations. To model the dynamics of a specific population, sub-models for $\beta$ and $\delta$ as functions of $t$ and $a$ and, in the case of nonlinear density dependence, the density $\rho$ itself must be specified. Gurtin and MacCamy [15] assume

where

$$
\beta=\beta(a, P), \quad \delta=\delta(a, P)
$$

$$
P=P(t)=\int_{0}^{+\infty} \rho(t, a) d a
$$

is total population size. Tucker and Zimmerman [29], in their generalization of Gurtin and MacCamy's results, allow for a more general functional dependence on $Q=Q(a, \rho(t, \cdot))$ (as well as a finite number of other structuring variables).

In order to develop models for populations in which there is a hierarchy based upon chronological age, we will assume instead that the vital rates $\beta$ and $\delta$ of an individual of age $a$ depend upon the number of individuals younger than (and/or older than) $a$, i.e. on the integrals

$$
Y=Y(t, a)=\int_{0}^{a} \rho(t, s) d s, \quad O=O(t, a)=\int_{a}^{+\infty} \rho(t, s) d s .
$$


Thus, in the McKendrick equations (1) and (2) we assume

$$
\beta=\beta(t, Y(t, a), O(t, a)), \quad \delta=\delta(t, Y(t, a), O(t, a))
$$

and the model equations become

$$
\begin{gathered}
\lim _{h \rightarrow 0} \frac{\rho(t+h, a+h)-\rho(t, a)}{h}=-\delta(t, Y(t, a), O(t, a)) \rho(t, a), \quad a>0, t>0 \\
\rho(t, 0)=\int_{0}^{+\infty} \beta(t, Y(t, a), O(t, a)) \rho(t, a) d a, \quad t>0 \\
\rho(0, a)=\varphi(a), \quad a \geqq 0 .
\end{gathered}
$$

Notice that we have also allowed for explicit time dependence in $\beta$ and $\delta$. Also notice that dependence on total population size $P(t)=Y(t, a)+O(t, a)$ is a special case. We do not, however, allow explicit dependence on $a$. Equations (6), (7), and (8) will be referred to as Problem 1.

The first goal of this paper is to prove an existence and uniqueness theorem for Problem 1. Under suitable smoothness assumptions

$$
\lim _{h \rightarrow 0} \frac{\rho(t+h, a+h)-\rho(t, a)}{h}=\partial_{t} \rho+\partial_{a} \rho
$$

(where $\partial_{t}=\partial / \partial t$ and $\partial_{\mathfrak{a}}=\partial / \partial a$ ) and (6) is a first order hyperbolic partial differential equation. Using the straight line characteristics of this equation, Gurtin and MacCamy transform the McKendrick equations (1)-(4) into an equivalent problem involving coupled integral equations to be solved for the two population level statistics $P(t)$ and $B(t)=\rho(t, 0)$ (the total population birth rate) for which they prove an existence/uniqueness result by means of the contraction principle. Tucker and Zimmerman also use this approach. The results of these authors do not directly apply to Problem 1, however, because of the kind of density dependence we have assumed in (5). On the other hand, Webb [31] uses a different approach to obtain a very general existence/uniqueness result which can be applied to the Problem 1. Nonetheless, we choose to give a totally different proof of an independent existence/uniqueness theorem for this problem by taking a slightly different approach from that of Gurtin and MacCamy. We do this in order to take advantage of the special kind of density dependence that we have assumed in (5). By this approach it will be seen how the dynamics of total population size $P(t)$ can be described by a scalar ordinary differential equation. This result provides a powerful way to study the global asymptotic dynamics of the total population size $P$ and, in turn, of the age specific density distribution $\rho$ for these kinds of model populations.

The following technical assumptions will be made on the vital rates $\beta$ and $\delta$ as functions of their arguments in (5). Let $R_{+}=[0,+\infty)$.

$\mathbf{A}_{1}: \beta, \delta \in C^{0}\left(R_{+}^{3}, R_{+}\right)$and $\exists$ positive reals $\beta_{0}, \delta_{0}>0 \quad \beta(t, Y, O) \leqq \beta_{0}, \delta(t, Y, O) \geqq \delta_{0}$ $\forall(t, Y, O) \in R_{+}^{3}$.

$\mathbf{A}_{2}$ : the partial derivatives $\partial_{Y} \beta(t, Y, O), \partial_{0} \beta(t, Y, O), \partial_{Y} \delta(t, Y, O)$, and $\partial_{0} \delta(t, Y, O)$ exist and are continuous on $R_{+}^{3}$.

$\mathbf{A}_{3}: \varphi \in L^{1}\left(R_{+}, R_{+}\right)$.

We define a solution of Problem 1 on an interval $[0, T], 0<T<+\infty$, to be a function $\rho:[0, T] \times R_{+} \rightarrow R_{+}$for which $\lim _{h \rightarrow 0}(\rho(t+h, a+h)-\rho(t, a)) h^{-1}$ 
exists $\forall(t, a) \in[0, T] \times R_{+}, \quad \rho(t, \cdot) \in L^{1}\left(R_{+}\right) \forall t \in[0, T], \quad P(t)=\int_{0}^{+\infty} \quad \rho(t, a) d a \in$ $C^{1}\left([0, T], R_{+}\right)$, and $(6),(7)$, and (8) are satisfied for $(t, a) \in[0, T] \times R_{+}$. A solution on an interval $[0, T), 0<T \leqq+\infty$, is defined similarly.

\section{Existence and uniqueness}

The existence and uniqueness of a solution of Problem 1 will be approached by means of an alternative problem based upon an equivalent set of equations. Consider the following equations for $Y=Y(t, a)$ and $P=P(t)$ :

with side conditions

$$
\begin{gathered}
P^{\prime}=B(t, P)-D(t, P) \\
Y=Q(P, Y)
\end{gathered}
$$

$$
\begin{gathered}
P(0)=\int_{0}^{+\infty} \varphi(u) d u, \quad Y(0, a)=\int_{0}^{a} \varphi(u) d u, \\
Y(t, 0)=0
\end{gathered}
$$

where the right hand sides in (9) and (10) are defined as follows:

and

$$
\begin{aligned}
& B(t, P) \doteq \int_{0}^{P} \beta(t, u, P-u) d u \\
& D(t, P) \doteq \int_{0}^{P} \delta(t, u, P-u) d u
\end{aligned}
$$

$$
Q(P, Y) \doteq\left\{\begin{aligned}
\int_{0}^{t} B(u, P(u)) \exp \left(-\int_{0}^{t-u} \tilde{\delta}(Y, P)(u+\alpha, \alpha) d \alpha\right) d u & \\
+\int_{0}^{a-t} \varphi(u) \exp \left(-\int_{0}^{t} \tilde{\delta}(Y, P)(\tau, u+\tau) d \tau\right) d u, & a \geqq t>0 \\
\int_{t-a}^{t} B(u, P(u)) \exp \left(-\int_{0}^{t-u} \tilde{\delta}(Y, P)(u+\alpha, \alpha) d \alpha\right) d u, & 0<a<t
\end{aligned}\right.
$$

where

$$
\tilde{\delta}(Y, P)(t, a) \doteq \delta(t, Y(t, a), P(t)-Y(t, a)) .
$$

Equations (9)-(12) will be referred to as Problem 2. We will show that under appropriate conditions a solution of Problem 2 yields a solution of Problem 1 and vice versa. By a solution of the Problem 2 on an interval $[0, T], 0<T<+\infty$, is meant a pair of functions $Y \in C^{0}\left([0, T] \times R_{+}, R_{+}\right), P \in C^{1}\left(R_{+}, R_{+}\right)$that satisfy (9)-(12) for $(t, a) \in[0, T] \times R_{+}$. A solution on an interval $[0, T), 0<T<+\infty$, is defined similarly.

In order to establish the relationship between Problems 1 and 2 it will be necessary to make a further technical assumption on the initial population density $\varphi$. One such assumption is

$\mathbf{A}_{4}: \varphi(a)$ has compact support on $R_{+}$. 
While this assumption is certainly reasonable from a biological point of view, it precludes the use of mathematically simple functions for $\varphi$, such as exponential functions. Another assumption that will serve our purposes is

$\mathbf{A}_{5}: \varphi \in C^{1}\left(R_{+}, R_{+}\right)$and $\lim _{t \rightarrow+\infty} \varphi(a)=0$.

The following result is proved in Appendix A.

Lemma 1. Assume $\mathbf{A}_{1}, \mathbf{A}_{2}, \mathbf{A}_{3}$ and either $\mathbf{A}_{4}$ or $\mathbf{A}_{5}$ hold. If the pair $Y(t, a), P(t)$ is a solution of Problem 2 on an interval $[0, T], T>0$, then $\partial Y(t, a) / \partial a$ exists and is continuous for $a \geqq t \in[0, T]$ and for $0<a<t \in[0, T]$ and $\rho \doteq \partial Y / \partial a$ solves Problem 1 on $[0, T]$. Conversely, if $\rho$ is a solution of Problem 1 on an interval $[0, T], T>0$, then $Y \doteq \int_{0}^{a} \rho(t, u) d u$ and $P \doteq \int_{0}^{+\infty} \rho(t, u) d u$ solve Problem 2 on $[0, T]$.

In general $\rho \doteq \partial Y / \partial a$ has a jump discontinuity across the characteristic $t=a$ (unless a compatibility condition on $\varphi$ holds); cf. [15], [29].

A global existence/uniqueness theorem for the Problem 2, and hence Problem 1, can be proved by the contraction mapping principle. This is done in Appendix B. The result is contained in the following theorem.

Theorem 2. Assume $\mathbf{A}_{1}, \mathbf{A}_{2}, \mathbf{A}_{3}$ and either $\mathbf{A}_{4}$ or $\mathbf{A}_{5}$ hold. Let $\left[0, T_{m}\right), 0<T_{m} \leqq+\infty$, be the maximal interval of existence for the solution $P(t)$ of the ordinary differential equation (9) under the initial condition (11). Then Problem 2, and hence Problem 1, has a unique solution on $\left[0, T_{m}\right)$.

Notice that this theorem asserts the existence of a solution on the whole interval $\left[0, T_{m}\right)$, and not just on some small interval containing 0 .

\section{Asymptotic dynamics}

In the important autonomous case when the vital rates are explicitly independent of time

$$
\beta=\beta(Y, O), \quad \delta=\delta(Y, O),
$$

Theorem 2 implies that the dynamics of the total population size $P(t)$ are governed by the scalar autonomous ODE

where

$$
P^{\prime}=f(P)
$$

$$
\begin{gathered}
f(P) \doteq B(P)-D(P) \\
B(P) \doteq \int_{0}^{P} \beta(u, P-u) d u, \quad D(P) \doteq \int_{0}^{P} \delta(u, P-u) d u .
\end{gathered}
$$

Therefore, the global dynamics of $P(t)$ are easily determined from the root properties of $f(P)$. In general, $P(t)$ is monotonic (hence non-oscillatory) and, as $t \rightarrow+\infty$, is either unbounded (i.e. approaches $+\infty$ ) or approaches one of the roots of $f(P)=0$ depending on the initial condition $P(0)=\int_{0}^{+\infty} \varphi(u) d u$.

We can also determine the asymptotic dynamics of the density $\rho(t, a)$. Suppose that $P(t) \rightarrow P_{\infty}$ as $t \rightarrow+\infty$. Consider the solution of $y_{\infty}(a)$ the scalar autonomous initial value problem

$$
y^{\prime}=\int_{y}^{P_{\infty}} \delta\left(u, P_{\infty}-u\right) d u, \quad y(0)=0 .
$$


Since $\delta>0$, it follows that

$$
y_{\infty}(a) \rightarrow P_{\infty} \text { monotonically as } a \rightarrow+\infty \text {. }
$$

Finally, for $a \geqq 0$ define

$$
\rho_{\infty}(a) \doteq B\left(P_{\infty}\right) \exp \left(-\int_{0}^{a} \delta\left(y_{\infty}(\alpha), P_{\infty}-y_{\infty}(\alpha)\right) d \alpha\right) .
$$

The proof of the following result appears in Appendix C.

Theorem 3. Assume, in addition to $\mathbf{A}_{1}, \mathbf{A}_{2}, \mathbf{A}_{3}$ and either $\mathbf{A}_{4}$ or $\mathbf{A}_{5}$, that the birth and death rates are autonomous in time as in (13). Then, for the unique solution $\rho(t, a)$ of Problem 1, the total population size $P(t)$ either increases monotonically without bound as $t \rightarrow T_{m}$ - or monotonically approaches an equilibrium of (14) (i.e. a root $P_{\infty} \geqq 0$ of $f(P))$ as $t \rightarrow+\infty$. In the latter case

$$
Y(t, a)-y_{\infty}(a) \rightarrow 0 \quad \text { and } \quad \rho(t, a)-\rho_{\infty}(a) \rightarrow 0 \quad \text { as } t \rightarrow+\infty
$$

uniformly for a restricted to any compact subinterval of $R_{+}$.

The global asymptotic dynamics of $P(t)$ for the autonomous Problem 1 are determined by the relatively simple scalar autonomous ordinary differential equation (14). Such general global results can be similarly derived in other interesting cases as well. As an example, suppose that the dependence of the birth and death rates on time $t$ is periodic, as might be the case when seasonal or other environmental oscillations are taken into account. The total population size is then governed by the periodic ordinary differential equation (9) from which one can draw similar global conclusions about the asymptotic dynamics of $P(t)$ (e.g. see [19], Part II). For example, if $P(t)$ is bounded then it must approach a periodic solution of (9) (which necessarily exists).

As another example, the explicit time dependence in $\beta$ and $\delta$ could be through a dependence upon a dynamic variable governed by its own differential equation. For example, we could have $\beta=\beta(R(t), Y, O)$ and $\delta=\delta(R(t), Y, O)$ where $R(t)$ is a resource or prey species whose dynamics are governed by an ordinary differential equation of the form $R^{\prime}=g(R, P)$. Then the model equations for the total population size and in resource are

where

$$
\begin{aligned}
& R^{\prime}=g(R, P) \\
& P^{\prime}=f(R, P)
\end{aligned}
$$

$$
f(R, P) \doteq \int_{0}^{P} \beta(R, u, P-u) d u-\int_{0}^{P} \delta(R, u, P-u) d u
$$

whose analysis is amenable to all the powerful techniques available for plane autonomous systems.

\section{Some applications}

In this section we give several applications of the results above and, in particular, of Theorem 3. In each case, a complete description of the global asymptotic dynamics of the model equations is obtained by analyzing a scalar ordinary differential 
equation or a planar system of ordinary differential equations. The primary purpose of these applications is to illustrate how a global asymptotic analysis can be carried out by using the methods developed above. Therefore, these applications and the biological issues involved will not be studied in depth.

\subsection{Contest and scramble competition}

There are many ways that the individuals in population can interact in order to produce self regulation, including various forms of competition for resources, mates, nesting sites, etc. and even intra-specific predation or cannibalism. Intraspecific competition can occur in many ways and has been categorized (sometimes ambiguously) in a variety of ways by biologists. Exploitative, interference, contest, and scramble competition are the most common classifications that are made in the literature. The effects of competition are felt by individuals and individuals typically differ in their response to competition. Individuals also differ in their ability to compete for resources with other individuals. Therefore, models that in some way take into account the differences between individuals, such as agestructured or size-structured models, will be the most effective in accounting for the dynamical effects of intra-specific competition. Of the many ways in which intraspecific competition can take place some of those that have been studied by mathematical models include juvenile vs. adult competition [6], [7], [9], [10], [23], [24], [28], effects on juvenile growth rate and size at maturation [8], [11], and resource availability based upon hierarchical rankings of individuals [17], [21] and [22]. Typically these studies focus on the dynamical consequences of various means of competition and consider their stabilizing or destabilizing effects on the population's dynamics.

It is often found that dominance rankings of individuals exist within populations which to a large extent determine an individual's access to resources (as well as mates, nesting sites, etc.). These rankings can be based upon physiological attributes such as age or body size or on sociological phenomena. It has been asserted in some studies that such rankings are generally a stabilizing influence on the population's dynamics [21] (although in [17] it is argued that if the "rewards" of dominance are too great then the effect of the ranking can be destabilizing).

The relationship between intra-specific competition based upon an agestructured ranking and the hierarchical age structured models considered above can be seen through Lomnicki's definition of scramble and contest competition ([21], p. 107). Suppose that the individuals of a population must compete for a limited resource. Scramble competition is defined to be the competitive situation in which each individual of the population can alter the resource consumption of every other individual. At the other extreme, contest (or asymmetric) competition occurs when a ranking hierarchy is in place with the property that the resource intake of an individual cannot be altered by individuals of lower rank in the hierarchy, although it can be altered by those of higher rank. One of Lomnicki's main conclusions is that contest competition is a more "resilient" or "stable" interaction than is scramble competition. In our first application we will briefly investigate this question by using a hierarchical age-structured competition model.

In this model it will be assumed that birth and death rates are a function of resource intake. "Pure" scramble and contest competition in our model occur when the resource intake and hence these vital rates of an individual of age $a$ depend on 
$P(t)=Y(t, a)+O(t, a)$ and $O(t, a)$, respectively. The dynamics of the resource will not be explicitly modeled (as in the next application). As pointed out by Lomnicki, scramble and contest competition modes are extreme cases and it is more realistic to expect that populations engage in both types of competition to one extent or another. One way (but certainly not the only way) to model such a case is to suppose that the age specific birth and death rates in (13) depend upon a weighted average $\gamma Y+(1-\gamma) O, \gamma \in[0,1]$, of the sub-population sizes $Y(t, a)$ and $O(t, a)$. In this case, $\gamma=0$ implies that only older individuals effect the vital rates of an individual of age $a$ and hence corresponds to contest competition, while $\gamma=1 / 2$ implies that the vital rates depend on total population size $P=Y+O$ and hence corresponds to scramble competition. Thus, we can use the weighting factor $\gamma$ as a measure of the amount of scramble competition occurring in the population. We accordingly restrict our attention to the interval $0 \leqq \gamma \leqq 1 / 2$.

Consider, then the birth and death rates

$$
\beta=\beta_{0} b(\gamma Y+(1-\gamma) O), \quad \delta=\delta_{0} d(\gamma Y+(1-\gamma) O)
$$

where the density dependence is expressed through the nonlinear functions $b$ and $d$ with the following properties (which guarantee that $\mathbf{A}_{1}$ and $\mathbf{A}_{2}$ hold):

$$
\begin{gathered}
b \in C^{2}\left(R, R_{+}\right), b(0)=1, b^{\prime} \leqq 0, b^{\prime \prime} \geqq 0, b(+\infty)=0 \\
d \in C^{2}\left(R, R_{+}\right), d(0)=1, d^{\prime} \geqq 0, d^{\prime \prime} \leqq 0 \\
\left(b^{\prime}\right)^{2}+\left(d^{\prime}\right)^{2} \neq 0,\left(b^{\prime \prime}\right)^{2}+\left(d^{\prime \prime}\right)^{2} \neq 0 \\
\beta_{0}, \delta_{0} \in R_{+} \text {and } \gamma \in[0,1 / 2] .
\end{gathered}
$$

The monotonicity assumptions on $b, d$ imply that increased population density has the deleterious effects of lowering birth rates and raising death rates. The functions $b, d$ are normalized by $b(0)=1, d(0)=1$ so that the constants $\beta_{0}, \delta_{0}$ are inherent birth and death rates, i.e. the birth and death rates in the absence of density dependence (e.g. at low population levels). The concavity assumptions on $b$ and $d$ are technical assumptions needed in the proofs of the results below (see Appendices D and E).

Applying the theorems above we find that the long term asymptotic dynamics of the population are determined by the scalar autonomous ODE (14), which can be written

where

$$
P^{\prime}=P F(P, \gamma)
$$

$$
F(P, \gamma) \doteq\left\{\begin{array}{cc}
\beta_{0} b\left(\frac{P}{2}\right)-\delta_{0} d\left(\frac{P}{2}\right) & \text { when } \gamma=1 / 2 \\
\frac{1}{(1-2 \gamma) P} \int_{\gamma P}^{(1-\gamma) P} \Delta(u) d u & \text { when } 0 \leqq \gamma<1 / 2
\end{array}\right.
$$

and

$$
\Delta(u) \doteq \beta_{0} b(u)-\delta_{0} d(u)
$$

Equation (17) is a scalar autonomous ordinary differential equation for $P$. Therefore, its global asymptotic dynamics can be completely determined by the root 
structure of the right hand side $P F(P, \gamma)$, which depends upon $\gamma$ [19]. Towards this end it can be shown that per capita growth rate $F(P, \gamma)$ has the properties (see Appendix D)

$$
F(0, \gamma)=\beta_{0}-\delta_{0}, \quad \frac{\partial}{\partial P} F(P, \gamma)<0, F(+\infty, \gamma)<0
$$

These properties imply that (17) has a positive equilibrium $P_{\infty}>0$ if and only if $n>1$ (in which case it is unique and stable) where $n$ is defined by

$$
n \doteq \frac{\beta_{0}}{\delta_{0}}
$$

This ratio is called the inherent net reproductive number; it is the expected number of offspring per individual per life time at low population densities. Since (17) is a scalar ODE we can immediately conclude from Theorem 3 the following global results for this intra-specific competition model.

Theorem 4. Consider a population modeled by (6)-(8) (i.e. Problem 1) with submodels for the vital rates given by (16) and an initial distribution $\varphi(a)$ satisfying $\mathbf{A}_{3}$ and $\mathbf{A}_{4}$ or $\mathbf{A}_{5}$. Assume that the initial total population size is nonzero, i.e. that $P(0)=\int_{0}^{\infty} \varphi(a) d a>0$. If $n \leqq 1$ then the population goes to extinction, i.e. the total populations size $P(t) \rightarrow 0$ and the distribution $\rho(t, a) \rightarrow 0$ (uniformly on compact age a intervals) as $t \rightarrow+\infty$. On the other hand, if $n>1$ then the population approaches a positive equilibrium or, more specifically, $P(t) \rightarrow P_{\infty}=P_{\infty}(\gamma)>0$ monotonically and $\rho(t, a) \rightarrow \rho_{\infty}(a)$ uniformly on compact age intervals as $t \rightarrow+\infty$ (where $\rho_{\infty}(a)$ is as described in Theorem 3).

One way to study differences between scramble and context competition is to investigate the dependency of the stable positive equilibrium $P_{\infty}(\gamma)>0$ on the parameter $\gamma \in[0,1 / 2]$ when $n>1$. The following result is proved in Appendix $\mathrm{E}$.

Theorem 5. Suppose $n>1$ in Theorem 4. Then the globally stable and attracting positive equilibrium $P_{\infty}(\gamma)$, as a function of $\gamma \in[0,1 / 2]$, has a strict maximum at $\gamma=0$ (contest competition) and a strict minimum at $\gamma=1 / 2$ (scramble competition).

Thus, in our model population we find that scramble competition is less favorable than contest competition in the sense that the total population equilibrium level is minimized under pure scramble competition and maximized under pure contest competition. Criteria other than relative equilibrium levels could be used to compare scramble and contest competition as well. For example, one could compare equilibrium "resilience" under the two competitive modes, i.e. the magnitude of the linearized eigenvalue $\partial(P F(P, \gamma)) / \partial P$ at $P=P_{\infty}(\gamma)$ as a function of $\gamma$. We will not pursue this or other criteria here, however.

With regard to the effects of scramble and contest competition on individual age classes we restrict ourselves to one simple example. Suppose that the intraspecific competition is not lethal and in no way effects the death rate, so that $\delta=\delta_{0}>0$ is a constant (as will be the case, incidently, in the next application). Then $y(a)=\left(1-\exp \left(-\delta_{0} a\right)\right) P_{\infty}(\gamma)$ and $\rho_{\infty}(a)=\delta_{0} \exp \left(-\delta_{0} a\right) P_{\infty}(\gamma)$. It follows that in this case all age classes are decreased (by the same fraction) by an increase in $\gamma$. In particular, they are all minimized by scramble competition and maximized by contest competition. 


\subsection{Competition for a dynamic resource}

In the age-specific competition model of the previous section the dynamics of the limited resource were not explicitly modeled. In this section we consider a modification of the model that incorporates the effects that the population has on the dynamics of the resource itself. This application will again illustrate how the dynamics of the total population size can be decoupled from that of the age specific density, this time resulting in a plane autonomous system of ordinary differential equations.

Let the quantity of resource be denoted by $R$ and suppose that in the absence of competition individuals consume this resource at a rate $U(R)$ where

$$
U \in C^{1}\left(R, R_{+}\right), U(0)=0, U^{\prime}>0 .
$$

In the presence of competition suppose that the consumption rate is given by $U(R) c(\gamma Y+(1-\gamma) O)$ where the competition factor $c$ satisfies

$$
c \in C^{2}(R,[0,1]), c(0)=1, c^{\prime}<0, c^{\prime \prime}>0, c(+\infty)=0 .
$$

Here, as in the model of the previous section, we have assumed that the effect of intra-specific competition on an individual of age $a$ is a function of the weighted average $\gamma Y+(1-\gamma) O$.

If we assume that the birth rate is proportional to the amount of consumed resource and that the resource has a negligible effect on the death rate, then

$$
\beta=\omega U(R) c(\gamma Y+(1-\gamma) O), \quad \delta=\delta_{0}>0
$$

where $\omega$ is a resource-to-offspring conversion factor. These sub-models for the birth and death rates satisfy $\mathbf{A}_{1}$ and $\mathbf{A}_{2}$. As in Sect. 5.1 we allow $\gamma$ to range from $\gamma=0$ (contest) to $\gamma=1 / 2$ (scramble). It follows from (9) that total population size is governed by the ODE

where

$$
P^{\prime}=\omega U(R) C(P, \gamma)-\delta_{0} P
$$

$$
C(P, \gamma) \doteq \int_{0}^{P} c(\gamma u+(1-\gamma)(P-u)) d u
$$

or

$$
C(P, \gamma)= \begin{cases}c\left(\frac{1}{2} P\right) P & \text { for } \gamma=1 / 2 \\ \frac{1}{1-2 \gamma} \int_{\gamma P}^{(1-\gamma) P} c(u) d u & \text { for } 0 \leqq \gamma<1 / 2\end{cases}
$$

If the amount of resource $R$ is held constant over time, then this model is just a special case of the one studied in the preceding Sect. 5.1. Suppose, however, that the consumption of the resource influences the amount of resource available at any given time so that $R=R(t)$ is a function of time. Specifically, if in the absence of the population the dynamics of the resource $R$ is governed by an equation $R^{\prime}=r(R)$, then in the presence of the population we have the equation

$$
R^{\prime}=r(R)-U(R) C(P, \gamma)
$$

for $R$. This equation and (22) constitute a plane autonomous system for the resource $R$ and the total population size $P$. 
As a specific example, consider the case when in the absence of the population the resource $R$ equilibrates exponentially to an equilibrium level $R_{0}>0$. A prototypical model for this situation is $r(R)=\left(R_{0}-R\right) r_{0}$ where $r_{0}>0$ is the exponential rate. Rescaling time and choosing the resource units appropriately we can assume without loss in generality that $r_{0}=R_{0}=1$ or that $r(R)=1-R$. The population level dynamics are then governed by the system of ordinary differential equations

$$
\begin{gathered}
R^{\prime}=1-R-U(R) C(P, \gamma) \\
P^{\prime}=\omega U(R) C(P, \gamma)-\delta_{0} P .
\end{gathered}
$$

It is possible to use the powerful techniques available for planar systems of ordinary differential equations to analyze and completely describe the global asymptotic dynamics of the system (24)-(25). The technical details are given in Appendix F. It turns out that the global dynamics again depend upon the inherent net reproductive number, which in this case is defined by

$$
n \doteq \frac{\omega U(1)}{\delta_{0}}>0 \text {. }
$$

$n$ is the expected number of offspring per individual over the course of its life span when the resource is held at its inherent equilibrium level $R=1$ and there is no intra-specific competition. The following result is proved in Appendix F.

Theorem 6. Consider a population modeled by (6)-(8) (i.e. Problem 1) with submodels for the vital rates given by (19)-(21) and an initial distribution $\varphi(a)$ satisfying $\mathbf{A}_{3}$ and $\mathbf{A}_{4}$ or $\mathbf{A}_{5}$. Assume that the initial total population size is nonzero, i.e. that $P(0)=\int_{0}^{\infty} \varphi(a) d a>0$. If $n<1$ then the population goes to extinction, i.e. the total population size $P(t) \rightarrow 0$, the resource $R(t) \rightarrow 1$, and the distribution $\rho(t, a) \rightarrow 0$ (uniformly on compact age a intervals) as $t \rightarrow+\infty$. On the other hand, if $n>1$ then the population approaches a positive equilibrium or, more specifically, $P(t) \rightarrow P_{\infty}=P_{\infty}(\gamma)>0, R(t) \rightarrow R_{\infty}(\gamma) \in(0,1)$, and $\rho(t, a) \rightarrow \delta_{0} \exp \left(-\delta_{0} a\right) P_{\infty}(\gamma)$ uniformly on compact age intervals as $t \rightarrow+\infty$.

It is shown in Appendix $F$ that the conclusions of theorem remain valid. That is to say, the total population equilibrium level $P_{\infty}(\gamma)$ is minimized at $\gamma=1 / 2$ and maximized at $\gamma=0$. The comments concerning the dependence of individual age classes on $\gamma$ made at the end of Sect. 5.1 apply in this case as well. Therefore, we again conclude that scramble competition is less favorable than contest competition in the same sense as in Sect. 5.1.

\subsection{Intra-specific predation}

Several recent modeling studies have addressed some of the dynamical consequences that have been attributed to intra-specific predation $[1],[4],[5],[18]$, [30]. These consequences include self regulation of population density [14], [26]; multiple stable equilibrium states and resulting hysteresis effects [1]; "life-boat" effects by which is meant that cannibalism allows a population to survive under circumstances when it would otherwise go extinct [26]; population oscillations [3], [26]; and periodic losses of entire age classes [26]. See [13], [14], and [26] for survey articles on the biological importance of intra-specific predation (or cannibalism). 
We will briefly consider here an age-structured model for a cannibalistic population in which older individuals may cannibalize younger individuals (but not the reverse). Cannibalism is primarily related to relative body sizes, with victims almost always being smaller than cannibals [26]. However, since body size often correlates well with chronological age, an age structured model may often be appropriate. Other age-structured models include those in [4], [18], [30]. A size-structured model similar to the age-structured model considered here can be found in [5].

Some of the dynamical consequences attributed to cannibalism that are mentioned above are due to the interplay between negative and positive feed-back effects on the population's vital rates, namely the negative feedback of population density on the death rate due to increased cannibalism and the positive feedback on the fertility (and survival) rate of cannibals due to the fact that victims serve as a food resource. To account for these effects we will introduce sub-models for fertility and mortality based upon both cannibalism and non-cannibalism food resource, following closely the models in [4] and [30].

We assume that the birth rate $\beta=\beta(E)$ of an individual of age $a$ is a function of its energy consumption rate $E$, which we decompose additively as $E=E_{n c}+E_{c}$ where $E_{n c}$ is the energy consumption rate obtained from non-cannibalistic resources and $E_{c}$ is that from cannibalism. If it is assumed that $E_{c}=E_{c}(Y(t, a))$ (since only individuals of age less than $a$ are potential victims), then $\beta$ becomes a function of $Y$ as required in Problem 1. We make a similar assumption about the noncannibalistic death rate $\delta_{n c}(E)$, which in addition to the death rate $\delta_{c}$ due to cannibalism, yield the total death rate $\delta=\delta_{n c}+\delta_{\mathrm{r}}$. Since we are assuming that only older individuals cannibalize an individual of age $a$, we write $\delta_{c}=\delta_{c}(O)$.

In summary we have the sub-models

$$
\beta=\beta(E(Y)), \quad \delta=\delta_{n c}(E(Y))+\delta_{c}(O), \quad E(Y)=E_{n c}+E_{c}(Y)
$$

in Problem 1 where

and

$$
\beta, \delta_{n c}, \delta_{c}, E_{c} \in C^{1}\left(R_{+}, R_{+}\right), \beta^{\prime}>0, \delta_{n c}^{\prime}<0, \delta_{c}^{\prime}>0, E_{c}>0
$$

$$
\beta(0)=0, \delta_{n c}(+\infty)>0, \delta_{c}(0)=0, E_{c}(0)=0 .
$$

These sub-models in (3) for Problem 1, which satisfy $\mathbf{A}_{1}$ and $\mathbf{A}_{2}$, lead us to the autonomous ordinary differential equation

$$
P^{\prime}=\int_{0}^{P}\left(\beta(E(u))-\delta_{n c}(P-u)-\delta_{0}(E(u))\right) d u
$$

for total population size $P=P(t)$.

As a specific example take $\beta=\omega E$ where $\omega>0$ is a conversion factor from consumed energy to offspring and $E_{c}=c_{1} Y, \delta_{c}=c_{2} O$ where the constants $c_{i} \geqq 0$ are "cannibalism coefficients". Then we get

where

$$
P^{\prime}=\delta_{n c}\left(E_{n c}\right)\left(n+\frac{c_{1}}{2 \delta_{n c}\left(E_{n c}\right)}\left(n-\frac{c_{2} E_{n c}}{c_{1} \delta_{n c}\left(E_{n c}\right)}\right) P\right) P-\int_{0}^{p} \delta_{n c}\left(E_{n c}+c_{1} u\right) d u
$$

$$
n \doteq \frac{\omega E_{n c}}{\delta_{n c}\left(E_{n c}\right)}
$$

is the inherent net reproductive number of the population in the absence of cannibalism. Except for notational changes this scalar ordinary differential 
equation is identical to one studied in [5] and therefore only some of its properties will be briefly described here.

In the absence of cannibalism $\left(c_{1}=c_{2}=0\right)$, equation (26) reduces to the linear equation $P^{\prime}=\delta_{n c}\left(E_{n c}\right)(n-1) P$ and hence the model population dies out exponentially if $n<1$ and grows exponentially if $n>1$. This exponential dynamics occurs because no density dependent regulation has been built into this model, except possibly for cannibalism.

When cannibalism is present $\left(c_{1}^{2}+c_{2}^{2} \neq 0\right)$, it is easy to see by linearization that the trivial equilibrium $P=0$ is (locally asymptotically) stable if $n<1$ and unstable if $n>1$. It is shown in [5] that the existence, uniqueness or non-uniqueness, and stability of positive equilibria depend on the concavity of the function $\delta_{n c}(E)$, the magnitude of the non-cannibalistic resource consumption rate $E_{n c}$, and the value of $n$. Possible cases for the existence and stability of positive equilibria, as a function of $n$, are graphically depicted in Figs. 1, 2. The cases (a), (b), and (c) in Fig. 1 occur for large, intermediate, and small non-cannibalistic energy consumption, respectively. Case (a) shows that cannibalism can be regulatory because, as we have seen, in the absence of cannibalism in this model the population grows without bound for $n>1$. Cases (b)-(c) show a "life-boat" effect where the population survives for some values of $n<1$, when it would go extinct without cannibalism. In Fig. 2 we see cases of multiple stable positive equilibria and hence hysteresis effects (via saddle-node bifurcations). For more details see [5].

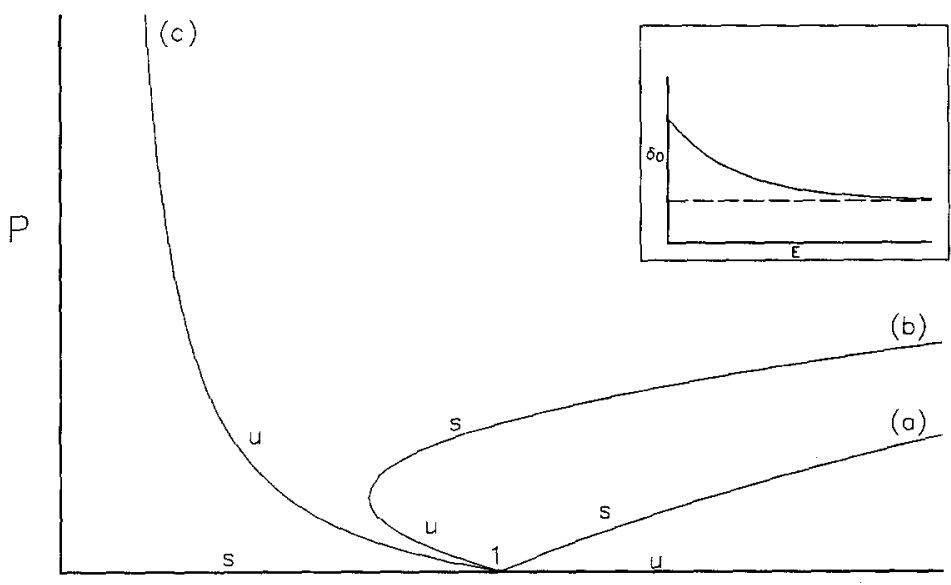

$n=$ inherent net reproductive number

Fig. 1. When cannibalism is absent in (31), the population goes extinct when $n<1$ and grows exponentially without bound when $n>1$. When cannibalism is practiced and the death rate $\delta_{0}(E)$ is concave upward as a function of the total energy consumption rate $E$ (as shown in the inset graph) there are three different kinds of bifurcation diagrams depicting the positive equilibria of (31) as functions of the inherent net reproductive number $n$. In case (a), which occurs for "large" non-cannibalistic energy consumption rates, positive equilibria exist for (and only for) $n>1$ and these equilibria are globally stable. This demonstrates that cannibalism can regulate population growth. For smaller non-cannibalistic energy consumption rates, cases (b) and (c) occur in which the population does not go to extinction for some values of $n<1$. This shows that it is possible for cannibalism to prevent extinction in circumstances when the population would otherwise go extinct 


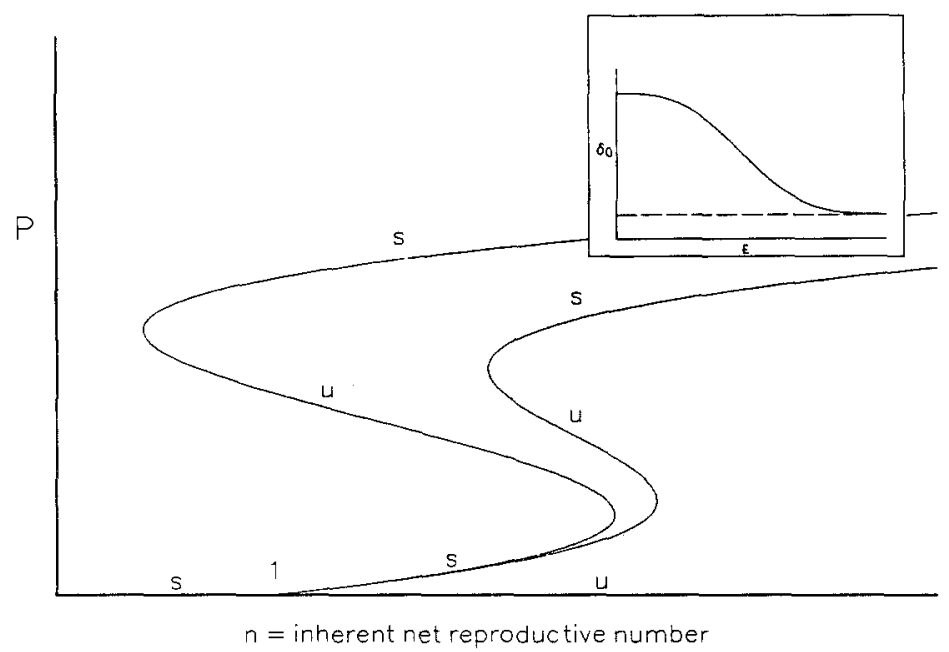

Fig. 2. When the death rate $\delta_{0}(E)$ is concave downward for small values of $E$ (as shown in the inset graph) double bends (i.e. saddle-node bifurcations) are possible in the equilibrium bifurcation diagrams for (31). This means that multiple, stable positive equilibrium states, and hence hysteresis effects, are possible for some values of $n$ (even for values of $n<1$ )

\section{Concluding remarks}

In this paper an age hierarchical population was defined as one in which the birth and death rates of an individual of age $a$ are dependent upon the size of the class of individuals younger than $a$ and/or the size of the class of individuals older than $a$. Under this assumption a proof of the existence and uniqueness of a solution of the McKendrick model equations was given which yields a decoupled ordinary differential equation of the total population size. Thus, for this class of model populations our results provide a means by which the population level dynamics can be related to individual (age specific) vital rates. Moreover, because the equation for total population size is a scalar ordinary differential equation, these results also provide the possibility that the global asymptotic dynamics of the population can be determined. For example, in the autonomous case Theorem 3 implies that only equilibrium dynamics are possible and shows how the asymptotic age distributions can be determined. The examples in Sect. 5 illustrate how these kinds of models can be used to address certain interesting problems concerning intra-specific competition and predation.

For general age-structured models it is not possible to derive a differential equation for total population size $P(t)$ that is decoupled from the equations for the density distribution $\rho(t, a)$. We succeeded in doing this in this paper because of the assumption (5), i.e. because of the assumption that the birth and death rates depend on age $a$ only through a dependence on the total densities $Y(t, a)$ and $O(t, a)$ of the sub-populations of younger and older individuals. Why this works mathematically is obscured in the technical details of the proofs in Appendix B. A heuristic explanation of the "trick" can be given as follows. Ignoring the technical questions that arise because of the discontinuity of $\rho(t, a)$ 
at $t=a$, we replace the limit in (1) by the expression $\partial_{t} \rho+\partial_{a} \rho$ and obtain the partial differential equation

$$
\partial_{t} \rho+\partial_{a} \rho+\delta(t, Y(t, a), O(t, a)) \rho=0
$$

If this equation is integrated from $a=0$ to $a=+\infty$ we arrive at the equation

$$
P^{\prime}(t)+0-\rho(t, 0)+\int_{0}^{+\infty} \delta(t, Y(t, a), O(t, a)) \rho(t, a) d a=0
$$

for $P(t)$ or using the birth equation (2)

$$
P^{\prime}(t)=\int_{0}^{+\infty} \beta(t, Y(t, a), O(t, a)) p(t, a) d a-\int_{0}^{+\infty} \delta(t, Y(t, a), O(t, a)) \rho(t, a) d a
$$

The "trick" that makes the decoupling work is based on the observation that $\rho(t, a)=\partial Y(y, a) / \partial a$ and hence this equation can be simplified to equation (9) by making the change of variable from $a$ to $u$ defined by $u=Y(t, a)$ (so that $\rho(t, a) d a=d u$ for fixed $t)$ in both of the integrals on the right hand side.

It is naturally to speculate on how far this trick might be extended to models of other kinds. For example, might it be possible to apply this or a similar trick to models in which the functionals $Y(t, a)$ and $O(t, a)$ in the crucial assumption (5) on the birth and death rates $\beta$ and $\delta$ are replaced by the weighted functionals

$$
\tilde{Y}(t, a)=\int_{0}^{a} a \rho(t, a) d a, \quad \tilde{O}(t, a)=\int_{a}^{+\infty} a \rho(t, a) d a ?
$$

A little reflection on the heuristic argument above (and variations such as first multiplying both sides of the partial differential equation by $a$ before integrating from $a=0$ to $a=+\infty$ ) shows the difficulty in trying to make the change of variable trick work in this case, at least with the amount of generality attained in this paper. However, further restrictive assumptions can sometimes make the trick work and produce decoupled equations for population level statistics. For example, suppose that the birth rate, in addition to a dependence on the weighted functionals $\widetilde{Y}, \widetilde{O}$, has a factor of $a$. That is to say, suppose that the per capita birth rate is proportional to age, so that

$$
\beta=\mathrm{a} \tilde{\beta}(t, \tilde{Y}(t, a), \widetilde{O}(t, a))
$$

Then

$$
\rho(t, 0)=\int_{0}^{+\infty} a \beta \rho d a=\tilde{B}(t, \tilde{P}(t))
$$

where

$$
\tilde{P}(t)=\int_{0}^{+\infty} a \rho(t, a) d a
$$


and $\widetilde{B}$ in is now defined in the same way $B$ was defined above except that $\tilde{\beta}$ is used in place of $\beta$. If in addition the death rate is constant

$$
\delta=\text { constant }>0
$$

then by integrating the partial differential equation (27) from $a=0$ to $a=+\infty$ and then integrating the equation obtained after a multiplication of both sides by $a$ from $a=0$ to $a=+\infty$, we get the two decoupled equations

$$
\begin{aligned}
P^{\prime} & =\widetilde{B}(t, \tilde{P}(t))-c P \\
\tilde{P}^{\prime} & =P-\delta \tilde{P}
\end{aligned}
$$

for $P$ and $\tilde{P}$.

This kind of trick with weighted functionals has also been successfully applied to size-structured hierarchical models in which resource consumption, and hence birth, death, and growth rates, are proportional to body size; see [5].

Some other extensions of the results and applications given above are possible. For example, the birth rate equation (7) can be replaced by

$$
\rho(t, 0)=\int_{0}^{t} g(t-s) \int_{0}^{+\infty} \beta(s, Y(s, a), O(s, a)) \rho(s, a) d a d s, \quad t>0
$$

in order to account for a gestation period. Here $g(s) d s$ is the probability that a birth occurs $s$ time units after conception. The theorems and proofs above can be straightforwardly modified to include this case, with (9) replaced by the scalar integrodifferential equation

$$
P^{\prime}=\int_{0}^{t} g(\tau) B(t-\tau, P(t-\tau)) d \tau-D(t, P(t))
$$

for total population size. In this case, non-equilibrium dynamics and oscillations are certainly possible because of the effects of the gestation delay.

Although the methods developed here are not directly applicable to interspecific interactions, it is possible to utilize them for interacting age hierarchical species which exploitatively compete for a common resource. For such a competition model a differential equation for the total population size of each species individually can be derived using the method and theorems proved here. This will be the subject of future research.

\section{Appendices}

\section{A Proof of Lemma 1}

Suppose that $\rho$ is a solution of Problem 1 on $[0, T)$. Then, following the argument of Gurtin and MacCamy [15], this is equivalent to $\rho$ satisfying the equations

$$
\rho(t, a)=\left\{\begin{array}{l}
\varphi(a-t) \exp \left(-\int_{0}^{t} \tilde{\delta}(Y, P)(\alpha, a-t+\alpha) d \alpha\right) \quad \text { for } a \geqq t \\
\rho(t-a, 0) \exp \left(-\int_{0}^{a} \tilde{\delta}(Y, P)(t-a+\alpha, \alpha) d \alpha\right) \quad \text { for } a<t
\end{array} .\right.
$$


From (7)

$$
\begin{aligned}
\rho(t, 0) & =\int_{0}^{\infty} \beta(t, Y(t, u), O(t, u)) \rho(t, u) d u \\
& =\int_{0}^{t} \beta(t, Y, P-Y) \frac{\partial Y}{\partial u} d u-\int_{t}^{\infty} \beta(t, P-O, O) \frac{\partial O}{\partial u} d u \\
& =\int_{0}^{t} \frac{\partial}{\partial u} \int_{0}^{Y} \beta(t, v, P-v) d v d u-\int_{t}^{\infty} \frac{\partial}{\partial u} \int_{0}^{o} \beta(t, P-w, w) d w d u \\
& =\int_{0}^{Y(t, t-)} \beta(t, v, P-v) d v+\int_{0}^{O(t, t+)} \beta(t, P-w, w) d w \\
& =\int_{0}^{Y(t, t-)} \beta(t, v, P-v) d v-\int_{P(t)}^{P(t)-o(t, t+)} \beta(t, v, P-v) d v \\
& =\int_{0}^{Y(t, t-)} \beta(t, v, P-v) d v+\int_{Y(t, t+)}^{P(t)} \beta(t, v, P-v) d v .
\end{aligned}
$$

Since $Y(t, a)$ is continuous in $a$ for all $t$ (even at $a=t$ ), we have $Y(t, t-)=Y(t, t+)$ and

$$
\rho(t, 0)=\int_{0}^{P(t)} \beta(t, v, P-v) d v=B(t, P(t)) .
$$

Using this in (28), replacing $a$ by $w$ and integrating $\rho(t, w)$ from $w=0$ to $w=a$, we obtain (10) (after a change of variable $u=\mathrm{t}-w$ ). Integrating instead from $w=0$ to $w=+\infty$, we obtain the integral equation

$$
\begin{aligned}
P(t)= & \int_{0}^{t} B(u, P(u)) \exp \left(-\int_{0}^{t-u} \tilde{\delta}(Y, P)(u+\alpha, \alpha) d \alpha\right) d u \\
& +\int_{0}^{+\infty} \varphi(u) \exp \left(-\int_{0}^{t} \tilde{\delta}(Y, P)(\tau, u+\tau) d \tau\right) d u
\end{aligned}
$$

for $\boldsymbol{P}(t)$. The assumption $\mathbf{A}_{4}$ or $\mathbf{A}_{5}$ insures that the second (improper) integral is uniformly convergent and consequently the smoothness assumption $\mathbf{A}_{1}$ guarantees that the right hand side of this equation is continuously differentiable with respect to $t$. A differentiation yields

$$
\begin{aligned}
P^{\prime}(t)= & B(t, P(t))-\int_{0}^{t} \tilde{\delta}(Y, P)(t, t-u) B(u, P(u)) \exp \left(-\int_{0}^{t-u} \tilde{\delta}(Y, P)(u+\alpha, \alpha) d \alpha\right) d u \\
& -\int_{0}^{+\infty} \varphi(u) \exp \left(-\int_{0}^{t} \tilde{\delta}(Y, P)(\tau, u+\tau) d \tau\right) \tilde{\delta}(Y, P)(t, u+t) d u \\
= & B(t, P(t))-\int_{0}^{t} \tilde{\delta}(Y, P)(t, u) B(t-u, P(t-u)) \\
& \times \exp \left(-\int_{0}^{u} \tilde{\delta}(Y, P)(t-u+\alpha, \alpha) d \alpha\right) d u
\end{aligned}
$$




$$
\begin{aligned}
& -\int_{t}^{+\infty} \tilde{\delta}(Y, P)(t, u) \varphi(u-t) \exp \left(-\int_{0}^{t} \tilde{\delta}(Y, P)(\tau, u+\tau-t) d \tau\right) d u \\
= & B(t, P(t))-\int_{0}^{t} \tilde{\delta}(Y, P)(t, u) \rho(t, u) d u-\int_{t}^{+\infty} \tilde{\delta}(Y, P)(t, u) \rho(t, u) d u .
\end{aligned}
$$

After manipulations as in (29) we obtain (9).

Conversely, supposed that the pair $Y(t, a), P(t)$ forms a solution of Problem 2. By the assumed smoothness of $\beta$ and $\delta$ we see from the right hand side of $(10)$ that $Y(t, a)$ has a partial derivative with respect to $a$ that is continuous on $0 \leqq a \leqq t$ and $t \leqq a$ (although it may have a jump discontinuity across $a=t$ ). Define $\rho(t, a) \doteq \partial Y(t, a) / \partial a$. A differentiation of (10) with respect to $a$ shows that this $\rho$ satisfies (28), and hence (6), (7), and (8). Moreover, it is clear from $\mathbf{A}_{1}$ and $\mathbf{A}_{3}$ that the right hand side of (10) converges as $a \rightarrow+\infty$. Hence the limit $Y(t,+\infty)=\lim _{a \rightarrow+\infty} Y(t, a)$ exists and satisfies (31) which, being equivalent to (9), implies that $Y(t,+\infty)=P(t)$ or in other words that $\int_{0}^{+\infty} \rho(t, a) d a=P(t)$. It follows that $\rho$ solves Problem 1 .

\section{$B$ Proof of Theorem 2}

We want to show that Problem 2 has a unique solution. Under assumption $\mathbf{A}_{2}$, there exists a unique solution $P(t)$ of (9) satisfying the initial condition $P(0)=$ $\int_{0}^{+\infty} \varphi(a) d a$ with a maximal interval of existence $\left[0, T_{m}\right)$. We need to show that $(10)$ has, for $P=P(t)$, a solution $Y$. This we will do by means of the contraction mapping principle.

For arbitrary but fixed $T \in\left[0, T_{m}\right)$ define $C_{T}^{0}=\left\{Y \in C^{0}\left([0, T] \times R_{+}, R\right)\right\}$. For any positive constant $\gamma>0, C_{T}^{0}$ is a Banach space under the norm $\|Y\|_{\gamma} \doteq \max _{[0, T] \times R_{+}}|Y(t, a)| e^{-\gamma t}$. The constant $\gamma$ will be chosen below. Define the compact subset

$$
S_{r}=\left\{Y \in C_{T}^{0}: 0 \leqq Y(t, a) \leqq r \quad \forall(t, a) \in[0, T] \times R_{+}\right\} .
$$

Denote $r_{T} \doteq T \beta_{0}+\int_{0}^{+\infty} \varphi(a) d a$.

First we observe from the definition of $Q(P, Y)$ and from assumptions $\mathbf{A}_{1}, \mathbf{A}_{3}$ that $0 \leqq Q(P, Y)(t, a) \leqq r_{T}$ for all $(t, a) \in[0, T] \times R_{+}$. Thus for $r \geqq r_{T}, Q(P, \cdot): S_{r} \rightarrow S_{r}$.

Finally we want to show that $Q(P, \cdot)$ is a contraction on $S_{r}$ with respect to $\|\cdot\|_{\gamma}$ provided that $\gamma>0$ is sufficiently large. Choose $Y_{1}, Y_{2} \in S_{r}$. Let $L_{T}$ be a Lipschitz constant for $\delta(t, Y, O)$ in $(Y, O)$ (independent of $t \in[0, T])$ on $\left[0, r_{T}\right] \times\left[0, P_{T}+r_{T}\right]$ where $P_{T}=\max _{[0, T]}|P(t)|$. The existence of $L_{T}$ is assured by $\mathbf{A}_{2}$. Then

implies

$$
\left|\delta\left(t, Y_{1}, O_{1}\right)-\delta\left(t, Y_{2}, O_{2}\right)\right| \leqq L_{T}\left(\left|Y_{1}-Y_{2}\right|+\left|O_{1}-O_{2}\right|\right)
$$

$$
\left|\delta\left(t, Y_{1}, P-Y_{1}\right)-\delta\left(t, Y_{2}, P-Y_{2}\right)\right| \leqq 2 L_{T}\left|Y_{1}-Y_{2}\right| .
$$

This, together with the fact that $\left|e^{-x}-e^{-y}\right| \leqq|x-y| \forall x, y \geqq 0$, implies for $0 \leqq a<t \leqq T$ that

$$
\begin{aligned}
\mid Q\left(P, Y_{1}\right)(t, a) & -Q\left(P, Y_{2}\right)(t, a) \mid \\
& \leqq 2 L_{T} \beta_{0} \int_{u=0}^{a} \int_{\alpha=0}^{u}\left|Y_{1}(t-u+\alpha, \alpha)-Y_{2}(t-u+\alpha, \alpha)\right| d \alpha d u
\end{aligned}
$$


and hence

$$
\begin{aligned}
& \left|Q\left(P, Y_{1}\right)(t, a)-Q\left(P, Y_{2}\right)(t, a)\right| e^{-\gamma t} \\
& \quad \leqq 2 L_{T} \beta_{0} e^{-\gamma t} \int_{u=0}^{a} \int_{\alpha=0}^{u}\left|Y_{1}(t-u+\alpha, \alpha)-Y_{2}(t-u+\alpha, \alpha)\right| e^{-\gamma(t-u+\alpha)} e^{\gamma(t-u+\alpha)} d \alpha d u \\
& \quad \leqq 2 L_{T} \beta_{0} e^{-\gamma t}\left\|Y_{1}-Y_{2}\right\|_{\gamma} \int_{u=0}^{a} \int_{\alpha=0}^{u} e^{\gamma(t-u+\alpha)} d \alpha d u \\
& \quad \leqq 2 L_{T} \beta_{0}\left\|Y_{1}-Y_{2}\right\|_{\gamma} \int_{u=0}^{a}(1 / \gamma) d u \\
& \quad \leqq\left(\frac{2 L_{T}}{\gamma}\right) \beta_{0} T\left\|Y_{1}-Y_{2}\right\|_{\gamma} .
\end{aligned}
$$

Similar inequalities for $0 \leqq t \leqq a, 0 \leqq t \leqq T$ lead to

$$
\begin{aligned}
& \left|Q\left(P, Y_{1}\right)(t, a)-Q\left(P, Y_{2}\right)(t, a)\right| e^{-\gamma t} \\
& \quad \leqq\left(\frac{2 L_{T}}{\gamma}\right)\left(\beta_{0} T+\int_{0}^{+\infty} \varphi(a) d a\right)\left\|Y_{1}-Y_{2}\right\|_{\gamma}
\end{aligned}
$$

and consequently

$$
\left|Q\left(P, Y_{1}\right)(t, a)-Q\left(P, Y_{2}\right)(t, a)\right| e^{-\gamma t} \leqq \frac{K_{T}}{\gamma}\left\|Y_{1}-Y_{2}\right\|_{\gamma}
$$

for all $(t, a) \in[0, T] \times R_{+}$where $K_{T}=2 L_{T}\left(\beta_{0} T+\int_{0}^{+\infty} \varphi(a) d a\right)$. It follows that

$$
\left\|Q\left(P, Y_{1}\right)-Q\left(P, Y_{2}\right)\right\|_{\gamma} \leqq \frac{K_{T}}{\gamma}\left\|Y_{1}-Y_{2}\right\|_{\gamma}
$$

and hence that $Q(P, \cdot)$ is a contraction on $S_{r}$ provided $\gamma$ is chosen larger than $K_{T}$.

This proves that Problem 2 has a unique solution on $[0, T]$ for every $T \in\left[0, T_{m}\right)$ and hence a unique solution on $\left[0, T_{m}\right)$.

\section{Proof of Theorem 3}

Since $P(t)$ satisfies the autonomous scalar ordinary differential equation (14), it follows immediately that its asymptotic dynamics are as described in the theorem. Suppose now that $P(t) \rightarrow P_{\infty}<+\infty, f\left(P_{\infty}\right)=0$, as $t \rightarrow+\infty$. From (10) for $t>a$ it is straightforward to compute

$$
\partial_{t} Y+\partial_{a} Y=B(P(t))-\int_{0}^{Y(t, a)} \delta(u, P(t)-u) d u .
$$

Here use is made of manipulations like those in (29). In characteristic coordinates $\tau=t-a, \alpha=a$ this equation becomes

$$
\partial_{\alpha} Y=B(P(\tau+\alpha))-\int_{0}^{Y} \delta(u, P(\tau+\alpha)-u) d u
$$


which is a scalar ordinary differential equation for $Y$ as a function of $\alpha$ with $\tau>0$ as a parameter. Using classical theorems on continuous dependence of solutions with respect to parameters, we can conclude that for $\alpha=a$ restricted to a compact interval the solution approaches that of the limit equation

$$
y^{\prime}=B\left(P_{\infty}\right)-\int_{0}^{y} \delta\left(u, P_{\infty}-u\right) d u
$$

as $t \rightarrow+\infty$. Since $f\left(P_{\infty}\right)=0$ implies $B\left(P_{\infty}\right)=D\left(P_{\infty}\right)=\int_{0}^{P_{\infty}} \delta\left(u, P_{\infty}-u\right) d u$, this equation is equivalent to (15). This proves $Y(t, a)-y(a) \rightarrow 0$ as $t \rightarrow+\infty$. Finally from (28) and (30) it follows that $\rho(t, a)-\rho_{\infty}(a) \rightarrow 0$ as $t \rightarrow+\infty$.

\section{$D$ Proof of Theorem 4}

First we note that the assumptions on $b, d$ imply

$$
\Delta(0)=\beta_{0}-\delta_{0}, \Delta^{\prime}(u)<0, \Delta^{\prime \prime}(u)>0, \Delta(+\infty)<0 .
$$

This implies that there exists a unique root $u^{*}>0$ of $\Delta(u)=0$. (See Fig. 3).

For $\gamma=1 / 2$ the equality and inequalities in (18) are obvious. To see that these hold for $0 \leqq \gamma<1 / 2$, we note that, by the mean value theorem for integrals, there exists a $\xi \in(\gamma P,(1-\gamma) P)$ such that

$$
\int_{\gamma P}^{(1-\gamma) P} \Delta(u) d u=\Delta(\xi)((1-\gamma) P-\gamma P)>\Delta((1-\gamma) P)(1-\gamma) P-\Delta(\gamma P) \gamma P
$$

and hence that

$$
\frac{\partial}{\partial P} F(P, \gamma)=\frac{\Delta((1-\gamma) P)(1-\gamma) P-\Delta(\gamma P) \gamma P-\int_{\gamma P}^{(1-\gamma) P} \Delta(u) d u}{(1-2 \gamma) P^{2}}<0 .
$$

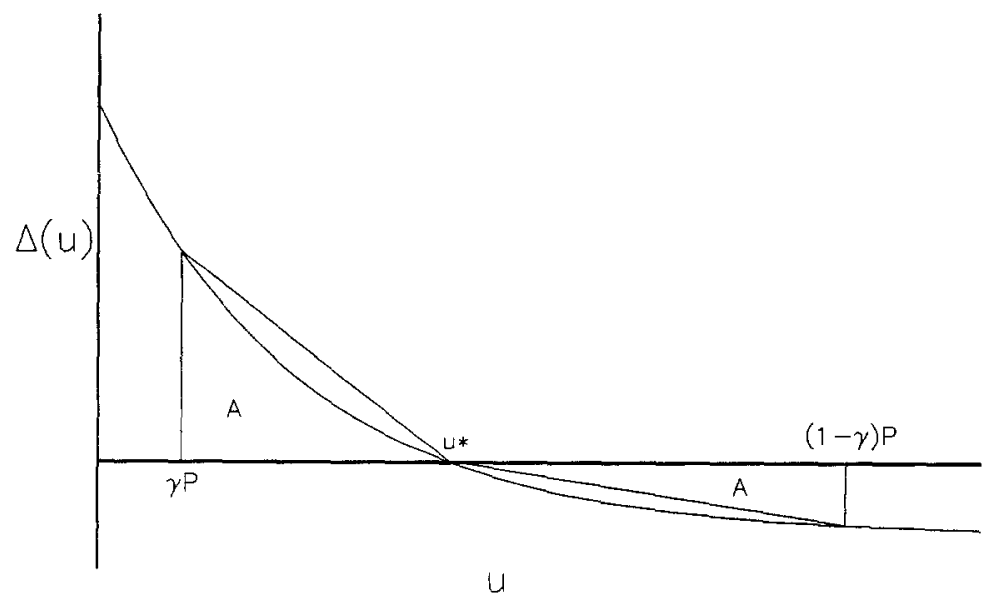

Fig. 3. The graph of the expression $\Delta(u)=\beta_{0} b(u)-\delta_{0} d(u)$ satisfying conditions (18) is shown, together with the secants relevant to the proof of $(20)$ 
Since the integrand $\Delta(u)<0$ for $u>u^{*}$, it follows that $F(+\infty, \gamma)<0$. Finally $F(0, \gamma)=\beta_{0}-\delta_{0}$ follows from an application of l'Hospital's rule.

\section{E Proof of Theorem 5}

From $f\left(P_{\infty}, \gamma\right)=0$ we have that $\partial P_{\infty} / \partial \gamma=-(\partial f / \partial \gamma)(\partial f / \partial P)$ and, since $\partial_{P} f=P_{\infty} \partial_{P} F\left(P_{\infty}, \gamma\right)<0$, we see that the sign of $\partial_{\gamma} P_{\infty}$ is the same as the sign of

$$
\frac{\partial}{\partial \gamma} f\left(P_{\infty}, \gamma\right)=-\frac{P_{\infty}}{1-2 \gamma}\left(\Delta\left((1-\gamma) P_{\infty}\right)+\Delta\left(\gamma P_{\infty}\right)\right) \text {. }
$$

We claim that

$$
\Delta\left((1-\gamma) P_{\infty}\right)+\Delta\left(\gamma P_{\infty}\right)>0 \text { for } 0 \leqq \gamma<1 / 2 .
$$

For $\beta_{0}>\delta_{0}$ the equilibrium equation implies $\int_{\gamma P}^{(1-\gamma) P} \cdot \Delta(u) d u=0$ and hence

$$
A \doteq \int_{\gamma_{P \infty}}^{u^{*}} \Delta(u) d u=-\int_{u^{*}}^{(1-\gamma) P_{\infty}} \Delta(u) d u
$$

where $u^{*}>0$ is the unique root of $\Delta(u)=0$. See Fig. 3. Define the positive constants $l_{1}=u^{*}-\gamma P_{\infty}$ and $l_{2}=(1-\gamma) P_{\infty}-u^{*}>0$.

Suppose $l_{2} \leqq l_{1}$. From the upward concavity assumption in (32), the slope of the tangent to the graph of $\Delta$ at the root $u^{*}$ is less negative than that of the secant between the points $\left(\gamma P_{\infty}, \Delta\left(\gamma P_{\infty}\right)\right)$ and $\left(u^{*}, 0\right)$; see Fig. 3 . Thus

$$
-\frac{\Delta\left(\gamma P_{\infty}\right)}{l_{1}}<\Delta^{\prime}\left(u^{*}\right)<\frac{\Delta\left((1-\gamma) P_{\infty}\right)}{l_{2}}
$$

which, together with $l_{2} \leqq l_{1}$, implies (33).

Suppose, on the other hand, that $l_{2}>l_{1}$. Again from the concavity of the graph of $\Delta$ it follows that the areas under the curves between these same pairs of points is less than that of the triangles determined by the secants; see Fig. 3. Hence

$$
\frac{1}{2} l_{1} A\left(\gamma P_{\infty}\right)>A>-\frac{1}{2} l_{2} \Delta\left((1-\gamma) P_{\infty}\right)
$$

which, together with $l_{2}>l_{1}$, implies (33).

Therefore, $\partial_{\psi} P_{\infty}<0$ on the interval $(0,1 / 2)$ and Theorem 5.2 follows.

\section{F Proof of Theorem 6}

We are, of course, only interested in non-negative solution of (24)-(25). A simple investigation of the direction field association with these equations on the coordinate axes shows that the positive quadrant $R_{+} \times R_{+}$is positively invariant (i.e. invariant forward in time). Moreover, all orbits in the positive quadrant are bounded forward in time. This can be seen as follows. Consider a right triangle formed by the positive coordinate axes and the hypotenuse $H: P=\omega(k-R)$, $0 \leqq R \leqq k$. We claim that such a triangle is positively invariant for all sufficiently large $k>0$. We need only check the direction field on the hypotenuse $H$, whose inwardly pointing normal has the direction given by the vector $(-\omega,-1)$. The flow on $H$ points inward if and only if the scalar product of the vector field defined by 
the right hand sides of (24)-(25) on $H$ with this inward normal is positive, i.e. if and only if

$$
\begin{gathered}
-\omega(1-R-U(R) C(P, \gamma))-\left(\omega U(R) C(P, \gamma)-\delta_{0} P\right) \\
=\omega\left(\left(1-\delta_{0}\right) R+\delta_{0} k-1\right)>0 \text { for } 0 \leqq R \leqq k .
\end{gathered}
$$

Since

$$
\omega\left(\left(1-\delta_{0}\right) R+\delta_{0} k-1\right) \geqq \begin{cases}\omega\left(\delta_{0} k-1\right) & \text { if } 1 \geqq \delta_{0} \\ k-1 & \text { if } 1<\delta_{0}\end{cases}
$$

we see that (34) holds provided $k>k_{0} \doteq \max \left\{1,1 / \delta_{0}\right\}$. Given any orbit in the positive quadrant we can construct a right triangle of this kind in such a way that the orbit remains inside for all $t \geqq 0$. This is done by choosing $k>k_{0}$ large enough so that the initial point of the orbit lies inside the triangle.

So far we have shown that any orbit of (24)-(25) starting initially in the positive quadrant remains in the positive quadrant and is bounded for all $t \geqq 0$. Next we note that the divergence of the vector field defined by (24)-(25) divided by $P$ is

$$
-\frac{1}{P}-U^{\prime}(R) \frac{C(P, \gamma)}{P}+\omega U(R)\left(\frac{C(P, \gamma)}{P}\right)^{\prime}
$$

which, because (20) implies $(C(P, \gamma) / P)^{\prime}<0$, is negative in the positive quadrant. It follows that there are no limit cycles in the positive quadrant (by the Dulac Negativity Criterion). This fact and the Poincare-Bendixson theorem will allow us to deduce the global asymptotic dynamics of (24)-(25) once we can account for all equilibria.

The equilibrium equations are

$$
\begin{gathered}
1-R-U(R) C(P)=0 \\
\omega U(R) C(P)-\delta_{0} P=0 .
\end{gathered}
$$

The only equilibrium on the coordinate axes is $(R, P)=(1,0)$. Any positive equilibria must satisfy the equivalent equations

$$
\begin{aligned}
& 1-\frac{U(1)}{n} P=R \\
& \frac{C(P, \gamma)}{P}=\frac{U(1)}{n U(R)}
\end{aligned}
$$

where

$$
n \doteq \frac{\omega U(1)}{\delta_{0}}>0
$$

(which biologically is the inherent net reproductive number of the population, or the expected number of offspring per individual over the course of its life span, when the resource is held at its inherent equilibrium level $R=1$ and there is no intra-specific competition). Thus, positive equilibria are determined by the positive roots of the equation

$$
\frac{C(P, \gamma)}{P}=\frac{U(1)}{n U(1-U(1) P / n)}
$$


on the interval $I: 0 \leqq P \leqq n / U(1)$. Given the definition (23) of $C(P, \gamma)$, it is easy to show that, for all $0 \leqq \gamma \leqq 1 / 2$, the left hand side of this equation is a continuously differentiable and decreasing function of $P$ on the interval $I$ which equals 1 when $P=0$. Since $U(R)$ is an increasing function of its argument, the right hand side of this equation is continuously differentiable and increasing function of $P$ which equals $1 / n$ when $P=0$ and approaches $+\infty$ as $P \rightarrow n / U(1)$. Therefore, there exists a positive root $P=P_{\infty}(\gamma)>0$ (and hence a positive equilibrium $R_{\infty}=\left(1-U(1) P_{\infty}(\gamma) / n, P_{\infty}=P_{\infty}(\gamma)\right)$ if and only if $n>1$, in which case it is unique.

The linearization of $(24)-(25)$ at the equilibrium $(R, P)=(1,0)$ has eigenvalues -1 and $\omega U(1)-\delta_{0}$. (Note: a straightforward calculations shows that $\partial C(0, \gamma)$ / $\partial P=1$.) Thus, this equilibrium is (locally asymptotically) stable if $n<1$ and unstable if $n>1$. In the latter case $(1,0)$ is a saddle whose stable manifolds lie on the $R$-axis and hence by the Hartman-Grobman theorem no positive orbit can have $(1,0)$ in its omega limit set. The Poincare-Bendixson Theorem now implies the global result for solutions of Problem 1 with vital rates given by (21) coupled with the resource equation (24).

Finally we consider how the equilibrium $P_{\infty}(\gamma)$ depends on $\gamma$. Noticing that

$$
\frac{\partial}{\partial \gamma}\left[\frac{C(P, \gamma)}{P}\right]=\frac{\int_{\gamma P}^{(1-\gamma) P} c(u) d u-\frac{(1-2 \gamma) P}{2}(c((1-\gamma) P)+c(\gamma P))}{\frac{P}{2}(1-2 \gamma)^{2}}
$$

and that the numerator is negative by the monotonicity and concavity assumptions on the function $c$ in (20) (the area under $c$ is less than that of the trapezoid formed by the secant), we see that as $\gamma$ increases the graph of the left hand side of (37) is lowered and hence the intersection point $P_{\infty}(\gamma)$ of with the graph of the right hand side is decreased. This means that the equilibrium level is minimized at $\gamma=1 / 2$ and maximized at $\gamma=0$.

\section{References}

1. Botsford, L. W:: The effects of increased individual growth rates on depressed population size. Am. Nat. 117, 38-63 (1981)

2. Busenberg, S. N., Iannelli, M.: Separable models in age-dependent population dynamics. J. Math. Biol. 22, 145-173 (1985)

3. Costantino, R. F., Desharnais, R. A.: Population Dynamics and the Tribolium Model: Genetics and Demography, Monographs on Theoretical and Applied Genetics 13. Berlin, Heidelberg, New York: Springer 1991

4. Cushing, J. M.: A simple model of cannibalism. Math. Biosci. 107(1), 47-71 (1991)

5. Cushing, J. M.: A size-structured model for cannibalism. Theor. Popul. Biol. 42(3), 347-361 (1992)

6. Cushing, J. M., Li, J.: On Ebenman's model for the dynamics of a population with competing juveniles and adults. Bull. Math. Biol. 51, 687-713 (1989)

7. Cushing, J. M., Li, J.: Juvenile versus adult competition. J. Math. Biol. 29, 457-473 (1991)

8. Cushing, J. M., Li, J.: Intra-specific competition and density dependent juvenile growth. Bull. Math. Biol. 54 (4), 503-519 (1992)

9. Ebenman, B.: Niche differences between age classes and intraspecific competition in agestructured populations. J. Theor. Biol. 124, 25-33 (1987)

10. Ebenman, B.: Competition between age classes and population dynamics. J. Theor. Biol. 131, $389-400(1988)$ 
11. Ebenman, B.: Dynamics of age- and size-structured populations: intraspecific competition, Size-Structured Populations, pp. 127-139 Ebenman and Persson (eds.). Berlin, Heidelberg, New York: Springer 1988

12. Ebenman, Bo, Persson, L: Size-Structured Populations: Ecology and Evolution. Berlin, Heidelberg, New York: Springer 1988

13. Elgar, M. A., Crespi, B. J.: Cannibalism: Ecology and Evolution among Diverse Taxa. Oxford: Oxford University Press 1992

14. Fox, Laurel, R.: Cannibalism in natural populations. Ann. Rev. Ecol. Syst. 6, 87-106 (1975)

15. Gurtin, M., MacCamy R. C.: Nonlinear age-dependent population dynamics. Arch. Rat. Mech. 54, 281-300 (1974)

16. Gurtin, M., MacCamy, R. C.: Some simple models for nonlinear age-dependent population dynamics. Math. Biosci. 43, 199-211; 213-237 (1979)

17. Gurney, W. S. C., Nisbet, R. M.: Ecological stability and social hierarchy. Theor. Popul. Biol. 16, 48-80 (1979)

18. Hastings, A.: Cycles in cannibalistic egg-larval interactions. J. Math. Biol. 24, 651-666 (1987)

19. Hale, J., Kocak, H.: Dynamics and Bifurcations, Texts in Applied Mathematics 3, Berlin, Heidelberg, New York: Springer 1991

20. Hoppensteadt, Frank: Mathematical Theories of Populations: Demographics, Genetics and Epidemics, Reg. Conf. Series in Appl. Math., SIAM, Philadelphia, 1975

21. Lomnicki, A: Population Ecology of Individuals, Monographs in Population Biology 25, Princeton University Press, Princeton, New Jersey, 1988

22. Lomnicki, A., Ombach, J.: Resource partitioning within a single species population and population stability: a theoretical model. Theor. Popul. Biol. 25, $21-28$ (1984)

23. Loreau, M.: Competition between age classes, and the stability of stage-structured populations: a re-examination of Ebenman's model, J. Theor. Biol. 144, 567-571 (1990)

24. May, R. M., Conway, G. R., Hassell, M. P., Southwood, T. R. E.: Time delays, density-dependence and single-species oscillations. J. Anim. Ecol. 43, 747-770 (1974)

25. Metz, J. A. J., Diekmann, O.: The Dynamics of Physiologically Structured Populations, Lecture Notes in Biomathematics 68. Berlin, Heidelberg, New York: Springer 1986

26. Polis, Gary, A.: The evolution and dynamics of intraspecific predation, Ann. Rev. Ecol. Syst. 12, 225-251 (1981)

27. Simmes, Stephen, D.: Age dependent population dynamics with non-linear interactions, Ph.D. dissertation, Carnegi-Mellon University Pittsburgh, 1978

28. Tschumy, W. O.: Competition between juveniles and adults in age-structured populations. Theor. Pop. Biol. 21, 255-268 (1982)

29. Tucker, S. L., Zimmerman, S. O.: A nonlinear model of population dynamics containing an arbitrary number of continuous structure variables, SIAM J. Appl. Math. 48(3), 549-591 (1988)

30. van den Bosch, F., de Roos, A. M., Gabriel, W.: Cannibalism as a life boat mechanism. J. Math. Biol. 26, 619-633 (1988)

31. Webb, G. F.: Theory of Nonlinear Age-dependent Population Dynamics. New York: Marcel Dekker, Inc. 1985

32. Werner, E. E., Gilliam, J. F.: The onogenetic niche and species interactions in size-structured populations. Ann. Rev. Ecol. Syst. 15, 393-425 (1984) 